Z Gerontol Geriat 2019 52 (Suppl 1):S1-S2 https://doi.org/10.1007/s00391-019-01508-4 Online publiziert: 28. Januar 2019

(c) Springer Medizin Verlag $\mathrm{GmbH}$, ein Teil von Springer Nature 2019

CrossMark

Hans Martin Hasselhorn ${ }^{1} \cdot$ Nico Dragano $^{2} \cdot$ Dirk Hofäcker $^{3} \cdot$ Morten Wahrendorf $^{2}$

'Lehrstuhl für Arbeitswissenschaft, Fakultät für Maschinenbau und Sicherheitstechnik, Bergische Universität Wuppertal, Wuppertal, Deutschland

${ }^{2}$ Institut für Medizinische Soziologie, Medizinische Fakultät, Heinrich-Heine-Universität Düsseldorf, Düsseldorf, Deutschland

${ }^{3}$ Professur für Methoden der quantitativen Sozialforschung, Institut für Soziale Arbeit und Sozialpolitik, Universität Duisburg/Essen, Essen, Deutschland

\title{
Soziale Ungleichheit beim Übergang von der Arbeit in den Ruhestand
}

eine Frage der Verteilung gesellschaftlicher Ressourcen mit hoher Relevanz für jeden Einzelnen handelt.

Das NRW-Forschungsnetzwerk „Arbeitsmarktteilhabe im höheren Erwerbsalter" [5] kam daher überein, ein wissenschaftliches Schwerpunktheft $\mathrm{zu}$ initiieren, in dem die Frage der sozialen Ungleichheit beim Ruhestandsübergang interdisziplinär wissenschaftlich thematisiert wird. Die Zeitschrift für Gerontologie und Geriatrie bietet hier auch aufgrund ihrer interdisziplinären Ausrichtung den passenden Rahmen. Zieldes Schwerpunktheftes ist, aktuelles Wissen zu bündeln und einen interdisziplinären Diskurs zur Thematik anzuregen.

Was ist „soziale Ungleichheit“? Sie liegt dann vor, „wenn die Zugehörigkeit zu einer sozialen Gruppe regelmäBig mit Vor- oder Nachteilen einhergeht “ [2]. Dies kann eine Frage des gesellschaftlichen „Oben“ und „Unten“ sein, wie Bildung oder sozialer Status (vertikale soziale Ungleichheit), aber nicht nur: Soziale Ungleichheiten finden sich genauso in Bezug auf Merkmale, die ein gesellschaftliches „Nebeneinander“ verschiedener Gruppen beschreiben, wie im Fall von Geschlecht und Migrationshintergrund (horizontale soziale Ungleichheit). Vielfach treten beide Formen sozialer Ungleichheiten gemeinsam in einem komplexen Wechselspiel miteinander auf [2].

Die in diesem Schwerpunktheft vorliegenden Beiträge bestätigen nicht nur die Breite und Vielfalt der Thematik, sondern verdeutlichen, dass die vielen beteiligten Disziplinen wie die Arbeitswissenschaft, Sozialepidemiologie, Gerontologie, Psychologie und Soziologie, jeweils originelle und sich ergänzende Sichtweisen - von der individuellen bis zur strukturell-politischen Ebene - beizusteuern haben. Unter Nutzung hochwertiger Daten betrachten sie unterschiedliche Aspekte horizontaler sozialer Ungleichheit (z.B. Geschlecht, Migrationsstatus, Beruf, Wirtschaftssektor, Nation) und auch vertikaler sozialer Ungleichheit (beruflicher Status, Einkommen). Dabei verbindet sie ihr jeweiliger Fokus auf die Unterschiedlichkeit der Rahmenbedingungen, des Erlebens und des Verhaltens der betrachteten sozialen Gruppen bei ihrem Übergangsprozess von der Arbeit in den Ruhestand.

Du Prel, Schrettenbrunner und Hasselhorn zeigen mit Daten der lidA-Kohortenstudie Unterschiede zwischen Gruppen horizontaler (Bildung) und vertikaler Ungleichheit (Geschlecht, Migrationsstatus) in Bezug auf ihre Motivation zum vorzeitigen Erwerbsausstieg: Demnach wollen geringer $\mathrm{Ge}$ bildete, Frauen und in niedrigen Bildungsschichten auch MigrantInnen der ersten Generation eher vorzeitig aus dem Erwerbsleben ausscheiden. Engstler belegt mittels prospektiver Daten des Deutschen Alterssurveys die hohe Umsetzungswahrscheinlichkeit von Erwerbsausstiegsplänen, wobei sich Be- 
schäftigtengruppen unterscheiden: Bei gering Qualifizierten findet er eine erhöhte Wahrscheinlichkeit für ungeplantes vorzeitiges Ausscheiden. Mäcken et al. zeigen auf Basis eines „Mixed-methods"-Ansatzes, dass ältere Beschäftigte im Gesundheitssektor zwar höhere Arbeitsbelastungen haben, sich aber in ihren Einstellungen zum Rentenübergang nicht von gleichaltrigen Beschäftigten anderer Sektoren unterscheiden. Weber et al. analysieren Erwerbstätigkeit nach Berentung bei Beschäftigten in den Niederlanden und finden hier ungünstigere Arbeitsbedingungen für Frauen.

In zwei Beiträgen werden die Auswirkungen politischer Paradigmenwechsel in Bezug auf soziale Ungleichheiten aus einer internationalen Perspektive thematisiert: Hofäcker, Hess und König dokumentieren mittels Daten des European Labour Force Survey verschiedene Spielarten dieser Paradigmenwechsel in vier europäischen Ländern. Die Freiwilligkeit der Ruhestandsentscheidung schwankt beträchtlich zwischen den untersuchten Ländern und ist dort am größten, wo ein konsistentes staatliches arbeitsmarkt- und sozialpolitisches Gesamtpaket zu finden ist (hier: Schweden und Österreich). Kadefors et al. zeigen unter Nutzung schwedischer Prozessdaten, dass die dortige Rentenreform des Jahres 2006 nicht nur zu einem starken Rückgang der Anzahl neuer Erwerbsminderungsrenten geführt hat (um 70\%), sondern kompensatorisch mit einem Anstieg an finanziell weniger attraktiven anderen Frühberentungsformen einherging; insbesondere „blue collar workers" haben diesen alternativen Ausstiegsweg vermehrt in Anspruch genommen.

Der Gerontologe David J. Ekerdt formulierte einst: „Retirement is the outcome of life chances" [3]. Diese Lebenslaufeffekte spiegeln sich in allen Beiträgen dieses Schwerpunktheftes, besonders pointiert aber in den drei Beiträgen wider, die Längsschnittdaten der Rentenversicherung nutzen, um Prädiktoren des Übergangs in die (gesundheitsbezogene) Erwerbsminderungsrente zu untersuchen. Die Studie von Götz, Dragano und Wahrendorf bestätigt ein erhöhtes Risiko für Erwerbs- minderung für Männer mit geringerer Bildung, geringerer beruflicher Position oder geringerem Einkommen. Bei Frauen waren die Effekte geringer, aber zumindest in der jüngeren Kohorte (45 bis 52 Jahre) ebenfalls deutlich ausgeprägt. Brzoska und Razum untersuchen die Inanspruchnahme einer medizinischen Rehabilitationsmaßnahme im Vorfeld einer Erwerbsminderungsrente und stellen fest, dass die Inanspruchnahme bei ausländischen Staatsangehörigen seltener und bei (Spät)AussiedlerInnen häufiger ist als bei Deutschen ohne Aussiedlerstatus. Nach Mika gehen der Erwerbsminderungsrente häufig unterbrochene Erwerbskarrieren sowie Phasen von Arbeitslosigkeit voran, was gleichzeitig maßgeblich die hierbei niedrigeren Rentenansprüche erklärt.

Der Gesundheit wird oft eine entscheidende Rolle für den Ruhestandsübergang eingeräumt [8]. Der soziale Gradient bezüglich Gesundheit ist bekannt (s. oben), allerdings hat sich die Forschung in der Untersuchung dieses Gradienten lange weitgehend auf Kinder, Jugendliche und das Erwerbsalter konzentriert. Lampert und Hoebel nutzen in ihrem Übersichtsbeitrag verschiedene Datenquellen, um zu untersuchen, wie sich die gesundheitliche Ungleichheit im Übergang vom mittleren zum höheren Lebensalter entwickelt. Auch im höheren Lebensalter finden sich hierzulande noch soziale Unterschiede in der Gesundheit, wenngleich diese etwas schwächer ausgeprägt sind als im mittleren Lebensalter.

Der Wunsch der Herausgeber dieses Schwerpunktheftes ist, in Zeiten immer länger werdender Erwerbsbiografien dazu beizutragen, dass sich die Diskussion zu Ruhestandsübergängen in Öffentlichkeit und Politik nicht zu sehr auf die Frage des (möglichst späten) Zeitpunkts des Erwerbsausstiegs beschränkt. Stattdessen sollte ebenso die angemessene Lebens- und Arbeitsqualität aller älteren Menschen während dieser Übergangsphase diskutiert werden und damit auch Fragen der Chancengleichheit, Gerechtigkeit und auch Würde in einer entscheidenden Lebensphase. Die hier zusammengestellten Beiträge zeigen, dass die Wissenschaft hier ihren Beitrag leisten kann.

\section{Korrespondenzadresse}

Prof. Dr. med. Hans Martin Hasselhorn Lehrstuhl für Arbeitswissenschaft, Fakultät für Maschinenbau und Sicherheitstechnik, Bergische Universität Wuppertal

Gaußstraße 20, 42119 Wuppertal, Deutschland hasselhorn@uni-wuppertal.de

Interessenkonflikt. H.M. Hasselhorn, N. Dragano, D. Hofäcker und M. Wahrendorf geben an, dass kein Interessenkonflikt besteht.

\section{Literatur}

1. Clemens W, Himmelreicher RK (2008) Erwerbsverlauf, Qualifikation und Rentenzugangsverhalten Eine Analyse mit Daten des Forschungsdatenzentrums der Rentenversicherung. Z Gerontol Geriatr 41:352-359. https://doi.org/10.1007/s00391008-0004-6

2. du Prel J, Schrettenbrunner C, Hasselhorn HM (2018) Vertikale und horizontale soziale Ungleichheit und Motivation zum vorzeitigen Erwerbsausstieg. Z Gerontol Geriatr 2018:2. https://doi.org/10.1007/s00391-018-1450-4

3. Ekerdt DJ (2010) Frontiers of research on work and retirement. J Gerontol B Psychol Sci Soc Sci 65B(1):69-80. https://doi.org/10.1093/geronb/ gbp109

4. Hasselhorn HM, Apt W (2015) Understanding employment participation of older workers: Creating a knowledge base for future labour market challenges. Research Report. BMAS/ BAuA, Berlin (120 Seiten). https://www.baua.de/ DE/Angebote/Publikationen/Kooperation/Gd81. pdf?_blob=publicationFile\&v=3. Zugegriffen: 04.01.2018

5. https://www.uni-due.de/biwi/hofaecker/ kompetenzcluster_nrw.php. Zugegriffen: 04.01.2018

6. Radl J (2013) Labour market exit and social stratification in western Europe: the effects of social class and gender on the timing of retirement Eur Sociol Rev 29(3):654-668

7. Radl J (2013b) Retirement timing and social stratification: a comparative study of labour market exit and Age norms in western Europe. Versita (De Gruyter Open), London

8. Van den HeuvelS, de Wind A (2015) Domain: health and health-related behaviour. In: Hasselhorn HM, Apt W (Hrsg) Understanding employment participation of older workers: creating a knowledge base for future labour market challenges. Research report.BMAS/BAuA, Berlin 